Gunkel: Ich war mit der „Meteor" vor ein paar Jahren im Persischen Golf. Dort herrscht ein starker Tankerverkehr, und an vielen Stellen wird Ol übernommen. Wir haben aber während der ganzen Zeit nicht ein einziges Mal einen Olfilm gesehen. Es wäre interessant, die Ursache herfür genauer zu untersuchen, denn sicherlich wird von den Tankern zumindest etwas OI über Bord gegeben bzw. werden sich geringfügige Leckagen in den Olleitungen finden, die dort Ladestationen versorgen. Ich glaube nicht, daß die hohe Wassertemperatur direkt verantwortlich ist, sondern der Verdacht liegt nahe, daß hier ein starker mikrobieller Abbau stattfindet.

REUTER: Dies kann auch daran liegen, daß die Schiffe vorher auf hoher See Schmutzöl von Bord gegeben haben. Denn wenn es dort in den Hafen kommt, muß es ja sauber sein, sonst bekommt es kein Ol. Vielleicht ist es auch eine Frage der Windverdriftung. Seeleute, die diese Route gefahren sind, behaupten, daß es dort schon $100 \mathrm{sm}$ vor einem Olhafen pestilenzartig stinken würde. Ihre Beobachtung verwundert mich; aber ich bin bei diesem Problem überfragt.

\title{
Ölbekämpfung und Bergung des Tankers „Anne Mildred Brøvig“
}

REUTER: Zunächst möchte ich darauf hinweisen, daß ein sehr umfangreicher Erfahrungsbericht der Wasser- und Schiffahrtsdirektion Hamburg ïber diesen Unfall existiert. Interessenten können ihn bei mir anfordern.

Etwa $40 \mathrm{sm}$ vor Helgoland wurde der 25000 -t-Tanker "Anne Mildred Brøvig" von dem Frachter "Pentland" gerammt. Die „Anne Mildred Brøvig" geriet in Brand, Ol strömte aus, das auf der Wasseroberfläche ebenfalls in Brand geriet. Durch die Tideströmung vertrieb das Wrack in Richtung Norden und versank bei einer Wassertiefe von $38 \mathrm{~m}$. Uber den Aufbauten befanden sich $22 \mathrm{~m}$ Wasser. Das Schiff hatte 30 Tanks von je 1000 bis $2500 \mathrm{t}$ Inhalt. Größere Olmengen traten insbesondere bei schlechtem Wetter aus, die deutlich sichtbar waren. Teilweise war die Ölschicht hinter dem Tanker unter $1 \mathrm{~mm}$ stark. Von mehreren Schiffen wurden in das austretende $O 1$ verschiedene Emulgatoren hineingespritzt. Insgesamt wurden 740001 Emulgatoren gesprüht, davon 40\% Moltoclar und 40\% Ascal Super 7-11. Außerdem wurden Slix, Gamlen, BP 1002 verwandt. Es waten noch größere Mengen an Emulgatoren vorrätig, die nicht mehr eingesetzt worden sind, nachdem die verhältnismäßig ungünstige Beurteilung dieser Bekämpfung durch Wissenschafter, insbesondere von der Bundesanstalt für Gewässerkunde, bekannt wurde. Außerdem hatten wir bemerkt, daß größere Schäden durch das O1 nicht eintraten. Den OOlaustritt aus dem Wrack zeigt Abbildung D2.

Das Wrack der "Anne Mildred Brøvig“ wurde mit dem größten Teil des noch in den Tanks befindlichen Ols geborgen. Hierbei hat sich die holländische Firma SmID sehr viel Ruhm erworben, die die schwierige Aufgabe mit sehr viel Geschick, Geduld und Tüchtigkeit löste. Sie hatte die Idee, das Achterschiff, das durch Explosion und Brand nur noch teilweise mit dem Vorschiff zusammenhing, durch eine Reihe von kleinen Haftladungen abzusprengen, um dadurch das Vorschiff auf seiner eigenen Ladung zum Aufschwimmen zu bringen. Vor der Sprengung wurde mit Hilfe des Pumpenschiffes "Gudrun Bonertz" begonnen, das Ladungsöl aus den Tanks in bereitstehende Tankleichter auszupumpen. Bei ruhiger See wurden Schlauchverbindungen zu den vorderen Tanks hergestellt (Abb. D3). Dieses Pumpmanöver ist jedoch nur etwa bis Windstärke 3 möglich. Trotz der damals für einige Tage herrschenden praktischen Windstille traten Wellen von 40 bis $50 \mathrm{~cm}$ Höhe auf. Die Tideströmung liegt etwa in der Größenordnung von $0,5 \mathrm{~m} / \mathrm{sec}$. In diesem Bereich wïrde ich es, wie schon ausgeführt, für ausgeschlossen halten, mit Olschlengeln $z \mathfrak{u}$ arbeiten. Die Bergung fand Ende April statt, und wir hatten glücklicherweise 8 bis 10 ruhige Tage. Zunächst wurden durch Taucher die Lüfter geschlossen. Nach der Sprengung schwamm das Vorschiff mit der Restladung auf; es hatte jedoch noch einen Tiefgang von $18 \mathrm{~m}$. Das Achterschiff liegt heute noch an dieser Stelle. Während dieser Arbeiten sind weitere, nicht unbeträchtliche Olmengen freigeworden. Das muß in Kauf genommen werden, wenn man auf diese Art und Weise die Gewässerverschmutzung durch die noch verbliebenen ca. 25000 t Ol vermeiden kann.

Das aufgeschwommene Wrack wurde nach Helgoland geschleppt, wobei auf dem ganzen Weg Preßluft auf die leergepumpten Tanks gedrückt wurde, um ein erneutes Sinken zu vermeiden. 
Im Windschatten von Helgoland, östlich der Düne, wurde das Leichtern fortgesetzt und es gelang, weitere $4500 \mathrm{t}$ in Tankleichter zu übernehmen. Das Wrack schwamm dadurch weiter auf. Wir waren sehr froh, daß das Leichtern ohne $Z$ wischenfälle verlaufen ist.

Auf hoher See fahren die Tanker mit geschlossenen Lüftern. Das Schiff darf aber im Revier, wo Kollisionsgefahr besteht, oder im Hafen nur entgast oder weitgehend entgast ankommen. Deshalb werden etwa 12 bis 24 Stunden vorher - die Vorschriften sind in den Ländern verschieden - die Lüfter geöffnet. Es war hier ein gewisses Verhängnis, daß die Lüfter bereits geöffnet waren, denn mindestens $1000 \mathrm{t}$ OOl sind durch die Lüfter ausgetreten. Andererseits hätte

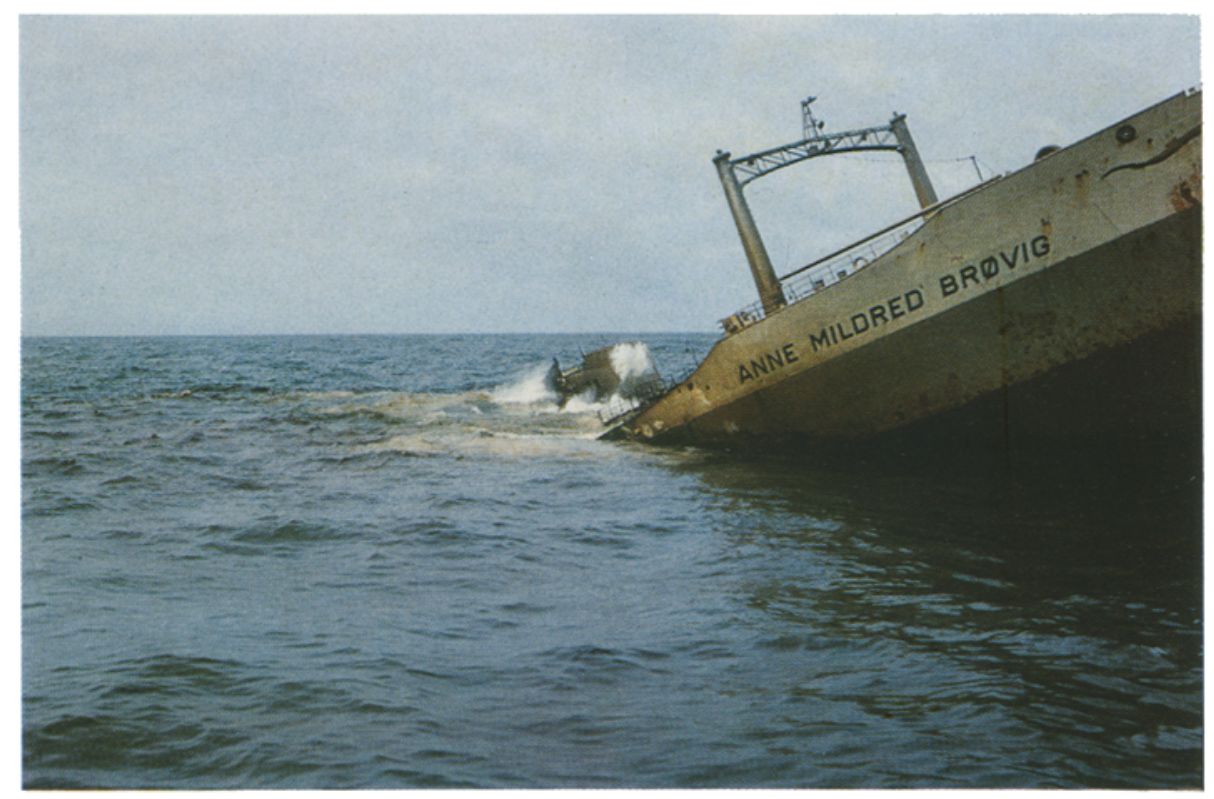

Abb. D2: Der Tanker „Anne Mildred Brøvig“ liegt mit dem Achterschiff auf Grund, während das Vorschiff auf der Ladung schwimmt. Der Olaustritt ist deutlich an der bräunlichen Färbung und der Beruhigung der Wellen zu erkennen (Photo: REUTER)

bei geschlossenen Lüftern, also nichtentgastem Schiff, bei der Kollision wesentlich mehr passieren können. Nach dem Leichtern hatte das Wrack 13,50 m bzw. 12,50 m Tiefgang. Um das Schiff auf ebenen Kiel zu bringen, wurde umgepumpt. Während dieser Aktion gingen noch geringe Mengen $O 1$ verloren, auf die Emulgatoren gespruht wurden. Anschließend wurde das Wrack der „Anne Mildred Brøvig" nach Wilhelmshaven geschleppt und in die große Schleuse, die 4. Einfahrt von. Wilhelmshaven, gebracht. Hier wurde dann der Rest der Ladung gelöscht.

Tomczak: Befindet sich noch OOl in den Tanks des Heckteils, der noch an der Unfallstelle liegt, und wann kommt das $O 1$ zum Vorschein?

REUTER: Wir beobachten das Achterschiff seitdern fortlaufend. In den ersten Tagen und Wochen, nachdem die Trennung vorgenommen worden ist, war noch ein ganz geringer Olaustritt festzustellen. Seit etwa einem Jahr ist keinerlei Olaustritt mehr beobachtet worden. Im Maschinenraum haben die Anfangsexplosionen stattgefunden, die Tagestanks etc. sind dabei verbrannt bzw. anschließend leergelaufen. Wir wußten, wie wenig Dieselöl in den sogenannten Tagestanks, aus denen die Motoren gefahren wurden, enthalten war. Dieses Ol ist mit Sicherheit verbrannt; das Achterschiff kann also kein $\mathrm{Ol}$ mehr abgeben. Es liegt dort auch jetzt sehr ruhig. Wir kontrollieren das Wrack in Abständen von 4 bis 8 Wochen, damit es bei Lage- 
veränderungen kein Schiffahrtshindernis wird. Ober dem Wrack sind jetzt etwa $25 \mathrm{~m}$ Wasser vorhanden.

BLoKker: Bei der "Torrey Canyon" hat man vielfach eine dicke Emulsion, das sogenannte "Chocolatemousse", beobachtet. Hat man das hier auch gesehen, oder ist nur eine dünne Schicht auf dem Wasser aufgetreten?

ReuTER: Ja; damit kommen wir zur Frage der Bekämpfung. Wir hatten ursprünglich gar keine Erfahrung. Der Grundsatz war, an der Austrittsstelle des Oles, also direkt am Wrack in die

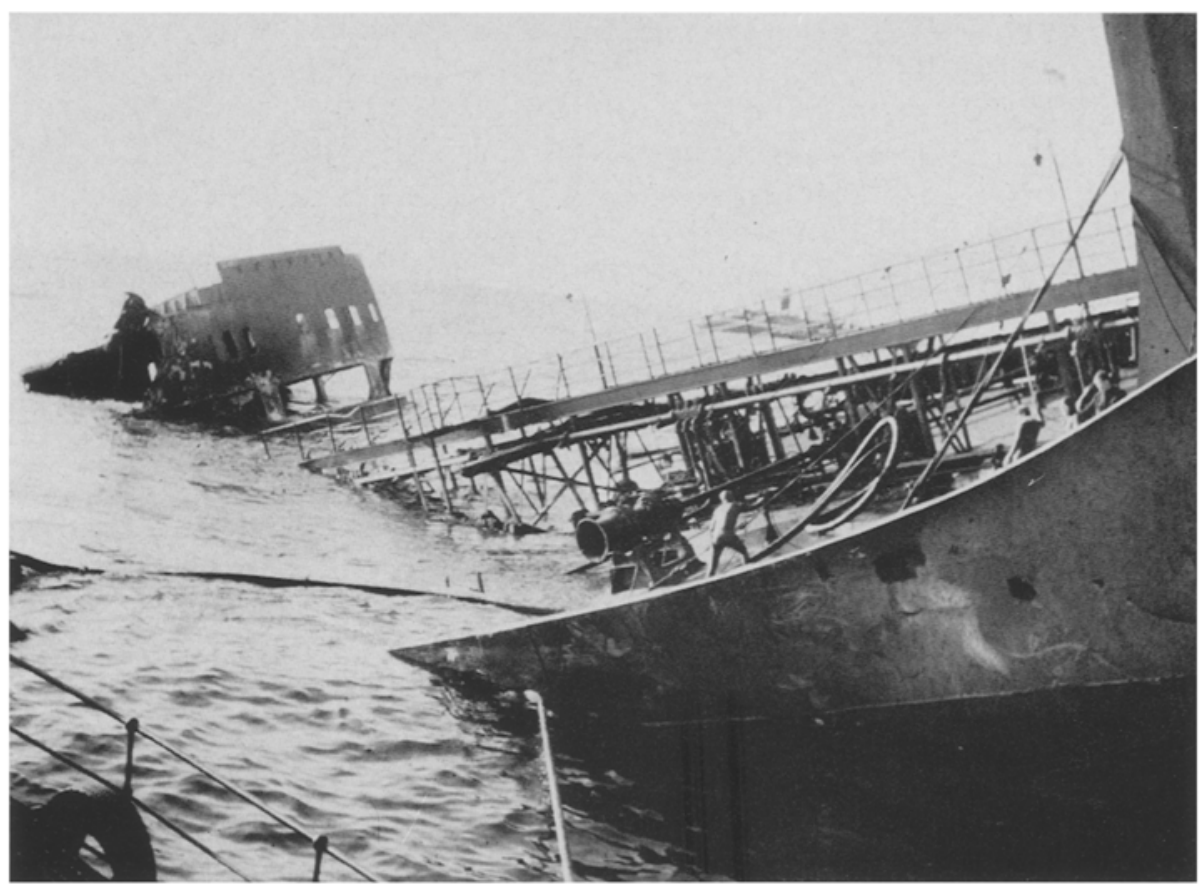

Abb. D3: Vom Pumpenschiff ist eine Schlauchverbindung zu den Ladungstanks im Vorschiff der "Anne Mildred Brøvig ce hergestellt worden (Photo: Reuter)

Schichten, die dort bis zu 20 bis $30 \mathrm{~cm}$, manchmal auch $40 \mathrm{~cm}$ stark waren, die Emulgatoren hineinzusprühen. Das haben wir einige Tage lang gemacht.

BLOKKER: Ich meine ohne Emulgatorzugabe. Bei der "Torrey Canyon" ist von allein eine sehr dicke Schicht dieser braun-gelben Masse entstanden. Hat man das auch hier beobachtet?

Reuter: Diese Schicht haben wir nur selten beobachtet. Größere Fladen von 2, 3 oder $4 \mathrm{qm}$ Größe haben wir schwimmen sehen. Wir haben teilweise versucht, auch diese zu bekämpfen. Eines unserer Schiffe hat diese Fladen über eine Strecke von 40 bis $50 \mathrm{sm} 2$ bis 4 Tage lang verfolgt und sie mehrfach mit Emulgatoren bespritzt. Sie haben sich aber nicht aufgelöst. Nachher haben wir sie aus der Sicht verloren.

Hellmann: Ist denn gar keine flache Olausbreitung auf Bruchteile von Millimetern oder noch darunter erfolgt?

Reuter: Wenn ruhige See war, dann trat so wenig Ol aus, daß es sich schnell weit verbreitete und nur einen feinen Film bildete, den wir nur in den ersten Tagen besprühten.

Gunkes: Der Olfilm war manchmal kaum als solcher zu erkennen; man stellte diese Stellen zunächst an der anderen Brechung und Form der Wellen fest. 
Reuter: Diese Stellen irisierten etwas. Vom Flugzeug aus konnte man sie gut sehen, weil die kleinen Windwellen dort nicht durchkamen.

Hellmann: Was hat die ganze Aktion ungefähr an Kosten verursadit?

REUTER: Wir haben für etwa 160000,- DM Emulgatoren gekauft. Ich darf übrigens den anwesenden Herren von der BP danken, daß sie uns 40000 Liter Emulgatoren kostenlos zur Verfügung gestellt haben. Ein Teil dieser Enulgatoren liegt noch in Cuxhaven für zukünttige Unglücksfälle greifbar. Die Kosten für die Schiffe miteingerechnet dürften eine Summe von etwa $600000,-D M$ ergeben haben. Darin ist aber der Verlust an Ol - ca. $15000 \mathrm{t}$ - nicht enthalten. Die restlichen $25000 \mathrm{t} \mathrm{O} 1$ mußten mit erheblichen Kosten aufgearbeitet werden. Ich habe mir berichten lassen, daß der Wert des Wracks und des Restöls größer war als die Kosten der Bergungsaktion; insofern hat sich audh finanziell die Bergung gelohnt.

\section{Bildung von Emulsionen und der Einsatz von Mineralölemulgatoren bei Verölungen}

Hellmann: Zur Einführung und Erklärung könnte man sagen: Milch ist eine Emulsion von Fett in Wasser. Man kann die Milch mit Wasser unbeschränkt verdünnen und kann so das Fett langsam zum „Verschwinden" bringen, wenn man nur genügend Wasser zugibt. Dieses Beispiel soll auch für die Olvernichtung herhalten. Man will durch Zugabe von gewissen Stoffen zum OI erreichen, daß es sich mit Wasser unbeschränkt vermischen läßt. Was die Natur bei der Milch vollbracht hat, ist technisch nicht so einfach. Jeder, der mit den Emulgatoren und Emulsionen zu tun gehabt hat, weiß, daß ganz bestimmte Bedingungen eingehalten werden müssen, daß es längst nicht genügt, einen bestimmten Stoff zum Ol zuzufügen und dann mit Wasser zu verdünnen. Die Firmen haben aber ihre Produkte unter dieser einfachen Voraussetzung lange Zeit angeboten. Wir haben bei allen untersuchten Produkten - es sind mehr als 20 gewesen - festgestellt, daß dieser Sachverhalt nicht so einfach ist, wie behauptet wurde. Die Emulgatoren sollen eine Vermischung des Oles mit dem Wasser erleichtern, d. h. sie müssen Eigenschaften des Wassers und des Oles besitzen. Die Emulgatoren setzen sich im wesentlichen aus einem organischen Lösungsmittel und einer aktiven Komponente, dem eigentlichen Emulgator, zusammen. Das Lösungsmittel hat verschiedene Funktionen zu erfüllen, die ich hier nicht näher erläutern möchte. Das Lösungsmittel madht aber volumenmäßig sehr viel des gesamten Produktes - bis zu $90 \%$ aus, so daß erklärlich ist, weshalb die Emulgatoren in starker Konzentration angewendet werden müssen. Unsere Versuche ergaben folgendes (vgl. Hellmann \& KNöpp 1967, HellmanN, KLEIN \& KNöpr 1966): Man kann eine Emulsion des Oles in Wasser nur erreichen, wenn man (1) einen Teil Ol auf einen Teil Emulgator gibt, (2) wenn die Kontaktzeit vor der Durchmischung genügend groß ist und (3) die Durchmischung O1/Emulgator/Wasser sehr intensiv ist. Dann kann man bei besonders guten Produkten eine Emulsion erhalten, die sich unbeschränkt mit Wasser vermischen läß $\mathrm{k}$, so daß man sagen kann, das Ol "verschwindet". Diese Versuche sind unter extrem günstigen Bedingungen in geschlossenen Systemen in Laboratorium durchgeführt worden, und zwar in einem Becherglas, in dem das OIl nicht ausweichen konnte, in dem aber das Ol immer wieder mit dem Emulgator reagieren mußte. Außerdem wurde bei 10000 bis 20000 Upm emulgiert. Wir haben versucht, die Ergebnisse auf die Natur zu übertragen und haben folgendes festgestellt: Es ist meistens nicht möglich, (1) eine ausreichende Menge Emulgator an das Ol heranzubringen, (2) die erforderliche Kontaktzeit Ol/Emulgator zu garantieren, weil das Wasser unter Umständen den Emulgator immer wieder herauswäscht und (3) eine ausreichende Turbulenz zu erzeugen, da das OOl bzw. die Ollache beim Mischen ausweicht. Auf die Schwierigkeiten, Riesenölfelder von allen Seiten zu besprühen und auf andere auftretende Schwierigkeiten möchte ich hier nicht zu sprechen kommen.

Gunkel: Sie sagten, Stabilität sei erst beim Verhältnis 1:1 erreicht. Gilt das für jedes Wasser? Sind die Verhältnisse im Süßwasser nicht günstiger?

Hellmann: Die Verhältnisse sind wechselnd und nicht voraussagbar; das Ergebnis hängt vom Emulgator ab. Es gibt Emulgatoren, die sich im Süßwasser günstiger und andere, die sich im Salzwasser günstiger auswirken (vgl. HellmanN \& KNöpp 1967). 